\title{
Stochastic mean-field formulation of the dynamics of diluted neural networks
}

\author{
D. Angulo-Garcia* \\ Consiglio Nazionale delle Ricerche (CNR), Istituto dei Sistemi Complessi, via Madonna del Piano 10, I-50019 Sesto Fiorentino, Italy \\ A. Torcini ${ }^{\dagger}$ \\ Consiglio Nazionale delle Ricerche (CNR), Istituto dei Sistemi Complessi, via Madonna del Piano 10, I-50019 Sesto Fiorentino, Italy \\ and INFN Sez. Firenze, via Sansone 1, I-50019 Sesto Fiorentino, Italy
}

(Received 29 September 2014; published 27 February 2015)

\begin{abstract}
We consider pulse-coupled leaky integrate-and-fire neural networks with randomly distributed synaptic couplings. This random dilution induces fluctuations in the evolution of the macroscopic variables and deterministic chaos at the microscopic level. Our main aim is to mimic the effect of the dilution as a noise source acting on the dynamics of a globally coupled nonchaotic system. Indeed, the evolution of a diluted neural network can be well approximated as a fully pulse-coupled network, where each neuron is driven by a mean synaptic current plus additive noise. These terms represent the average and the fluctuations of the synaptic currents acting on the single neurons in the diluted system. The main microscopic and macroscopic dynamical features can be retrieved with this stochastic approximation. Furthermore, the microscopic stability of the diluted network can be also reproduced, as demonstrated from the almost coincidence of the measured Lyapunov exponents in the deterministic and stochastic cases for an ample range of system sizes. Our results strongly suggest that the fluctuations in the synaptic currents are responsible for the emergence of chaos in this class of pulse-coupled networks.
\end{abstract}

DOI: 10.1103/PhysRevE.91.022928

PACS number(s): 05.45.Xt, 87.19.1j, 87.10.Mn, 05.40.Ca

\section{INTRODUCTION}

In pioneering studies devoted to excitatory pulse-coupled networks of leaky integrate-and-fire (LIF) neurons [1,2], Abbott and van Vreeswiijk have shown that these models in a globally coupled configuration can exhibit only two kinds of evolution, both regular. The first one, termed the splay state, is associated to collective asynchronous dynamics and the second one, called partial synchronization, corresponds to coherent periodic activity in the network. The latter regime is characterized by periodic oscillations in the neural activity and by quasiperiodic motions of the single-neuron membrane potentials [2]. The introduction of random dilution in such a network, achieved by considering an Erdös-Rényi distribution for the connectivity degrees, induces chaoticity in the system and fluctuations in the collective activity [3]. Fluctuations and chaos are due to the nonequivalence of the neurons in the network. However, for massively connected networks, where the average in-degree is proportional to the system size [4], the dynamics becomes regular in the thermodynamic limit, recovering the evolution of the globally coupled system [3,5]. On the other hand, for sparse networks, where the in-degree value is constant independently of the network size [4], the system remains chaotic even in the thermodynamic limit [6].

A fundamental question we would like to address in this paper is whether the effect of the frozen network heterogeneity can be reproduced in terms of a homogeneous model with additive noise. In particular, we are interested in reproducing the chaotic behavior observed in the diluted system. As homogeneous model we consider a fully coupled (FC) network

\footnotetext{
*david.angulo@fi.isc.cnr.it

†alessandro.torcini@cnr.it
}

displaying only regular motions and we focus on the partially synchronized regime, where the macroscopic variables are periodic [2]. The addition of noise to the membrane potential evolution induces irregular oscillations in the dynamics, observable both at the neuronal and at the macroscopic levels. To reproduce the dynamics of a specific deterministic diluted (DD) network, we employ as noise amplitudes in the stochastic model the ones measured in the original system. As a result, the stochastic model is able to mimic the main microscopic and macroscopic features of the original diluted system and even the chaoticity properties of the deterministic system. Furthermore, we are able to mimic the dynamics of networks composed by thousands of neurons by employing a stochastic model with only 100 elements.

Previous studies have been devoted to the dynamical equivalence among the effect induced by noise on a system of identical excitable units and by quenched disorder in the model parameters (diversity) [7,8]. In our analysis the diversity among neurons arises from the fact that each network element has a different (randomly chosen) set of connections. In particular, our study finds placement in the framework of the research works devoted to noise-induced chaotic dynamics [9-11]; however, we are now dealing with a high-dimensional system with a nontrivial collective behavior. Furthermore, our approach, despite being developed for a simple network model, can be easily extended to a large class of complex networks.

The paper is organized as follows. Section II A is devoted to the introduction of the DD model as well as of dynamical indicators able to characterize microscopic and macroscopic dynamics in this system. In the same subsection, the results concerning the dynamical evolution of deterministic FC and diluted networks are briefly revisited. In Sec. II B the stochastic model developed to mimic the dynamics of the diluted system is introduced. Three methods to estimate the Lyapunov 
spectrum in pulse-coupled neural networks are revised in Sec. III. In the same section the three methods are compared by applying them to deterministic systems. Furthermore, the generalization of two of such methods to stochastic pulse-coupled networks with white and colored noise is also presented. Section IV A deals with the analysis of the reconstructions of the microscopic and macroscopic features of the DD network via the stochastic approach. The Lyapunov analysis for the stochastic models is reported in Sec. IV B and the results are compared with the ones obtained for the corresponding DD systems. Finally, a summary and a brief discussion of the obtained results is reported in Sec. V together with a sketch of possible future developments.

\section{MODELS AND METHODS}

\section{A. Diluted deterministic network}

\section{The model}

We will focus our study on a diluted network of $N_{D}$ LIF, where the membrane potential $v_{i}$ of each neuron evolves according to the following first-order differential equation

$$
\dot{v}_{i}(t)=a-v_{i}(t)+g E_{i}(t) \quad i=1, \ldots, N_{D},
$$

where $a>1$ represents a suprathreshold dc current and $g E_{i}$ the synaptic current, with $g>0$ being the excitatory synaptic coupling. Whenever the membrane potential of the $i$-th neuron reaches a fixed threshold $v_{\Theta}=1$, the neuron emits a pulse $p(t)$ transmitted, without any delay, to all the postsynaptic neurons and its potential is reset to $v_{R}=0$. In particular, the field $E_{i}(t)$ is given by the linear superposition of the pulses $p(t)$ received at the previous spike times $\left\{t_{n}\right\}$ by the $i$-th neuron from the pool of its presynaptic neurons. In this paper, in analogy with previous studies $[1,2,12]$, we assume that the transmitted pulse is an $\alpha$ function, namely $p(t)=\alpha^{2} t \exp (-\alpha \mathrm{t})$, where $\alpha^{-1}$ is the width of the pulse. In this case, the evolution of each field $E_{i}(t)$ is ruled by the following second-order differential equation:

$$
\ddot{E}_{i}(t)+2 \alpha \dot{E}_{i}(t)+\alpha^{2} E_{i}(t)=\frac{\alpha^{2}}{K} \sum_{n \mid t_{n}<t} C_{j, i} \delta\left(t-t_{n}\right)
$$

where $C_{j, i}$ is a $N_{D} \times N_{D}$ random matrix whose entries are 1 if there is a synaptic connection from neuron $j$ to neuron $i$, and 0 otherwise, and $K$ is the number of presynaptic connections of the $i$-th neuron. For a FC network $K=N$ and all the fields are identical, since each neuron receives exactly the same sequence of spikes. By introducing the auxiliary variable $P_{i} \equiv \alpha E_{i}+\dot{E}_{i}$, the second-order differential equation (2) can be rewritten as

$$
\dot{E}_{i}=P_{i}-\alpha E_{i}, \quad \dot{P}_{i}=-\alpha P_{i}+\frac{\alpha^{2}}{K} \sum_{n \mid t_{n}<t} C_{j, i} \delta\left(t-t_{n}\right) .
$$

Therefore, the network evolution is ruled by the $3 N_{D}$ Eqs. (1) and (3) which can be exactly integrated between a spike event occurring at time $t_{n}$ and the successive one at time $t_{n+1}$, thus defining the following event-driven map $[3,13]$ :

$$
\begin{aligned}
& E_{i}(n+1)=E_{i}(n) e^{-\alpha \tau(n)}+P_{i}(n) \tau(n) e^{-\alpha \tau(n)}, \\
& P_{i}(n+1)=P_{i}(n) e^{-\alpha \tau(n)}+C_{m, i} \frac{\alpha^{2}}{K}, \\
& v_{i}(n+1)=v_{i}(n) e^{-\tau(n)}+a\left(1-e^{-\tau(n)}\right)+g H_{i}(n) .
\end{aligned}
$$

The $m$-th neuron is the next firing neuron, which will reach the threshold at time $t_{n+1}$, i.e., $v_{m}(n+1) \equiv 1$. One should notice that the event-driven map is an exact rewriting of the continuous time evolution of the system evaluated in correspondence of the spike emissions, therefore it can be considered a Poincare section of the original flux in the $3 N_{D}$ dimension. Indeed, the event-driven map is $3 N_{D}-1$ dimensional, since the membrane potential of the firing neuron is always equal to 1 in correspondence of the firing event. Here $\tau(n)=t_{n+1}-t_{n}$ is the time between two consecutive spikes, which can be determined by solving the implicit transcendental equation

$$
\tau(n)=\ln \left[\frac{a-v_{m}(n)}{a+g H_{m}(n)-1}\right],
$$

where the expression $H_{i}(n)$ appearing in Eqs. (4c) and (5) has the form

$$
\begin{aligned}
H_{i}(n)= & \frac{e^{-\tau(n)}-e^{-\alpha \tau(n)}}{\alpha-1}\left[E_{i}(n)+\frac{P_{i}(n)}{\alpha-1}\right] \\
& -\frac{\tau(n) e^{-\alpha \tau(n)}}{(\alpha-1)} P_{i}(n) .
\end{aligned}
$$

In this paper, we consider connectivity matrices $C_{j, i}$ corresponding to random graphs with directed links and a fixed in-degree $K$ for each neuron [14]. This amounts to have a $\delta$ distribution centered at $K$ for the in-degrees and a binomial distribution with average $K$ for the out-degrees. In particular, we examine massively connected networks, where the in-degree grows proportionally to the system size, namely our choice has been $K=0.2 \times N_{D}$. As we have verified, the main results are not modified by considering Erdös-Rényi distributions with an average in-degree equal to $K$. A few tests are also devoted to the dynamics of sparse networks, in particular this has been done by maintaining the in-degree constant to $K=100$ and by increasing the system size $N_{D}$ up to 10000 .

\section{Microscopic and macroscopic dynamical indicators}

In contrast to FC systems, the presence of dilution in the network induces fluctuations among the instantaneous values of the fields $\left\{E_{i}(t)\right\}[3,5]$. These fluctuations can be estimated by evaluating the instantaneous standard deviation $\sigma_{E}(t)$ of the individual fields $E_{i}(t)$ with respect to their instantaneous average value $\bar{E}(t)$, defined as follows:

$$
\bar{E}(t)=\frac{1}{N_{D}} \sum_{i=1}^{N_{D}} E_{i}(t),
$$

$$
\sigma_{E}(t)=\left[\frac{1}{N_{D}} \sum_{i=1}^{N_{D}} d_{i}(t)^{2}\right]^{1 / 2}
$$


where $d_{i}(t)=E_{i}(t)-\bar{E}(t)$ denotes the instantaneous fluctuation of the $i$-th field with respect to their average over the network. Similarly, we can define $\bar{P}$ and $\sigma_{P}$. Obviously, for a FC network $E_{i} \equiv \bar{E}, P_{i} \equiv \bar{P}$ and $\sigma_{E}=\sigma_{P} \equiv 0$. In the following, we will consider an unconstrained time average of the fluctuations $\left\langle\sigma_{E}\right\rangle$, as well as a conditional time average $\left\langle\sigma_{E}(\bar{E}, \bar{P})\right\rangle$ evaluated whenever the value of the average fields falls within a box of dimension $\Delta \bar{E} \times \Delta \bar{P}$ centered at $(\bar{E}, \bar{P})$.

To measure the level of correlation present in the field fluctuations $d_{i}(t)$, we measure the associated autocorrelation function

$$
C_{E}(\tau)=\frac{\left\langle\frac{1}{N_{D}} \sum_{i=1}^{N_{D}} d_{i}(t+\tau) d_{i}(t)\right\rangle}{\left\langle\left(\sigma_{E}(t)\right)^{2}\right\rangle},
$$

where $\langle\cdot\rangle$ indicates the average over time. The time interval over which the fluctuations are correlated can be estimated by measuring the decorrelation time $\tau_{d}$ from the the initial decay of $C_{E}(\tau)$.

The collective activity in the network can be studied by examining the macroscopic attractor in the $(\bar{E}, \bar{P})$ plane as well as the distributions of the average fields $F(\bar{E})$ and $F(\bar{P})$. On the other hand, the microscopic dynamics has been characterized by considering the distribution $F$ (ISI) of the single-neuron interspike intervals (ISIs) as well as the associated first return map.

\section{Diluted versus fully coupled dynamics}

As already mentioned in the Introduction, the dynamical regimes observable for FC LIF networks, with postsynaptic potentials represented as $\alpha$ function, have been analyzed in Refs. [1,2]. These regimes are the so-called splay state and partial synchronization. The splay state is characterized by a constant value for the field $E$ and by a perfectly periodic evolution of the single neurons. On the other hand, in the partially synchronized regime, the common field reveals a perfectly periodic evolution, while the single-neuron dynamics is quasiperiodic [12]. In the present paper we will focus in the latter regime, where collective oscillations in the network activity are present, in this case the macroscopic attractor corresponds to a closed curve in the two-dimensional $(\bar{E}, \bar{P})$ plane. The introduction of random dilution in the system induces fluctuations $d_{i}$ in the fields $E_{i}$ with respect to their network average $\bar{E}$. Therefore the collective attractor still resembles a closed orbit, but it has now a finite width whose
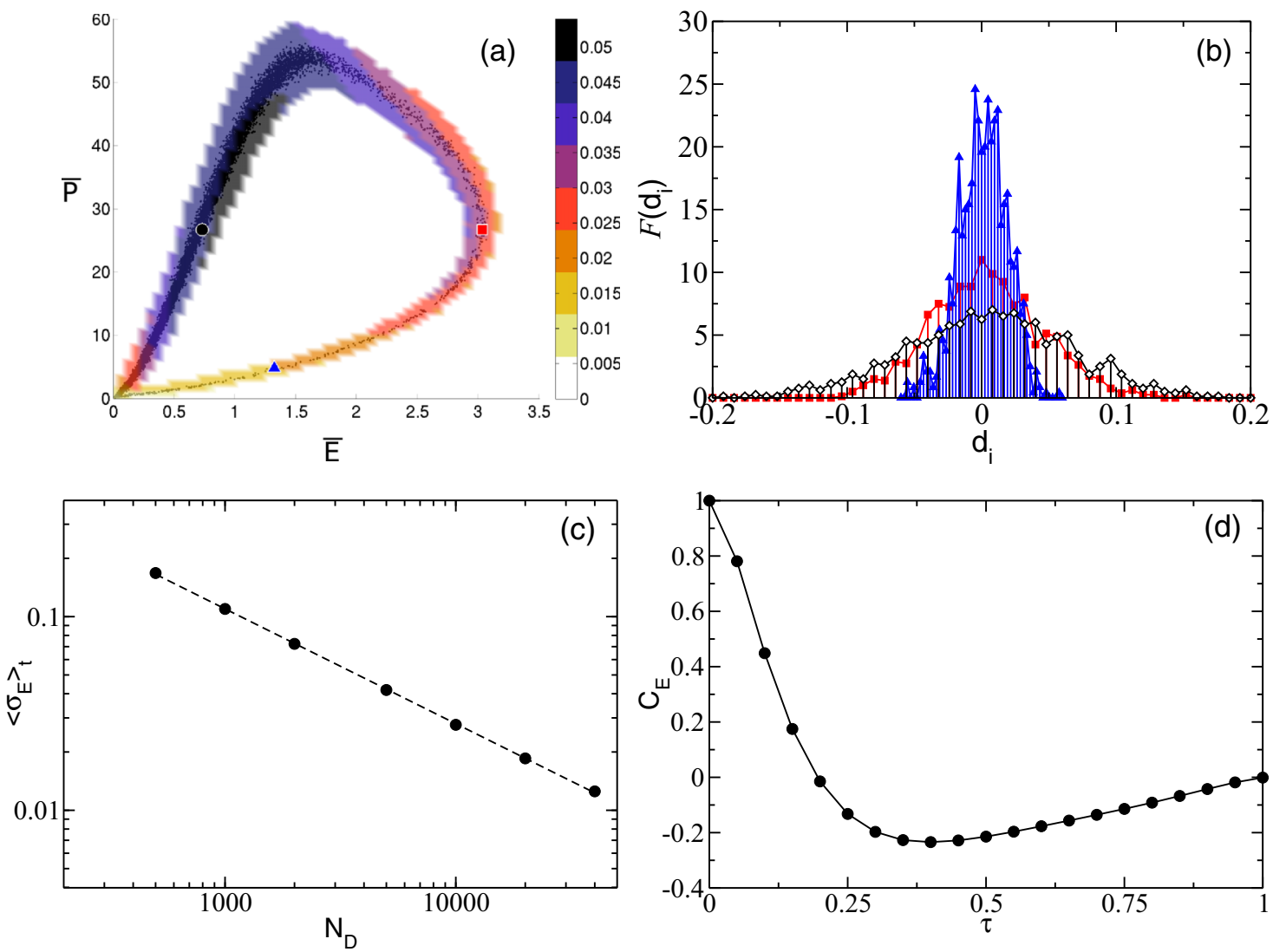

FIG. 1. (Color online) Characterization of the field fluctuations for a DD network. (a) Macroscopic attractor reported in the $(\bar{E}, \bar{P})$ plane (black dots); the colormap (superimposed on the attractor) quantifies the time-averaged values of the fluctuations $\left\langle\sigma_{E}(\bar{E}, \bar{P})\right\rangle$. These are estimated over a $100 \times 100$ grid with resolution $\Delta \bar{E}=0.06$ and $\Delta \bar{P}=0.8$. (b) PDFs $F\left(d_{i}\right)$ of the deviation from the average field $d_{i}$ estimated in three different points along the attractor. These points are indicated in panel (a) with the same symbol and color code. (c) Fluctuations of the fields $\left\langle\sigma_{E}\right\rangle$ averaged both in time and all along the whole attractor as a function of the system size $N_{D}$ (filled circles). The dashed line indicates a power-law fitting to the data, namely $\left\langle\sigma_{E}\right\rangle \propto N_{D}^{-0.6}$. (d) Autocorrelation function $C_{E}(\tau)$ of the fluctuations of the fields $d_{i}$. For all the reported data the parameters are fixed to $g=0.5, \alpha=9, a=1.05$, and $K=0.2 N_{D}$. The system size is set to $N_{D}=500$, apart in panel (c). The reported quantities have been evaluated over $10^{5}-10^{6}$ spikes, after discarding an initial transient of $10^{6}$ spikes. 
value depends on the values of $(\bar{E}, \bar{P})$ [see Fig. 1(a)]. As shown in Fig. 1(b), the fluctuations $d_{i}$ are approximately Gaussian distributed for any point $(\bar{E}, \bar{P})$ along the curve. Therefore, the $d_{i}$ can be characterized in terms of their standard deviation $\left\langle\sigma_{E}\right\rangle$ averaged in time; this quantity, as previously shown in Refs. [3,5], vanishes in the thermodynamic limit for massively connected networks. Indeed, this is verified also in the present case as shown in Fig. 1(c), thus indicating that for sufficiently large system sizes one recovers the regular motion observed for FC systems. It should be recalled that for sparse networks the fluctuations do not vanish, even for diverging system sizes [5]. Furthermore, the field fluctuations present a decorrelation time $\tau_{c} \simeq 0.1$, measured from the decay of the autocorrelation function $C_{E}(\tau)$ [see Fig. 1(d)], which is essentially independent from the system size, as we have verified.

Another relevant aspect of the diluted system dynamics is that the random dilution of the links renders the finite network chaotic. In particular, for a massively connected network the system becomes regular in the thermodynamic limit, while a sparse network remains chaotic even for $N_{D} \rightarrow \infty$ [12]. This result suggests that the degree of chaoticity in the system is related to the amplitude of the fluctuations $d_{i}(t)$ of the macroscopic fields.

\section{B. Fully coupled stochastic network}

The question that we would like to address is whether the dynamics of the DD network can be reproduced in terms of an equivalent FC network with additive stochastic terms. As a first approximation, we assume that the erratic dynamics of the DD system is essentially due to the field fluctuations $d_{i}(t)$. Therefore, we rewrote the dynamics of the diluted system as follows:

$$
\begin{aligned}
\dot{E}(t) & =P(t)-\alpha E(t), \\
\dot{P}(t) & =-\alpha P(t)+\frac{\alpha^{2}}{N_{S}} \sum_{n \mid t_{n}<t} \delta\left(t-t_{n}\right), \\
\dot{v}_{i}(t) & =a-v_{i}(t)+g E(t)+g \xi_{i}(t) \quad i=1, \ldots, N_{S},
\end{aligned}
$$

where each neuron is driven by the same mean-field term $E(t)$, generated by the spikes emitted by all the neurons, plus an additive stochastic term $\xi_{i}(t)$. Notice also that we use a different number of neurons in the reduced model $N_{S}<N_{D}$ since the asymptotic evolution of a FC system is fairly well retrieved already with a relatively small number of neurons. We will consider both white noise as well as colored one. In particular, for white Gaussian noise

$$
\left\langle\xi_{w_{i}}(t) \xi_{w_{j}}\left(t^{\prime}\right)\right\rangle=D^{2} \delta_{i, j} \delta\left(t-t^{\prime}\right)
$$

with a zero average value, namely $\left\langle\xi_{w_{i}}\right\rangle=0$. For colored noise, we considered exponentially time correlated noise as follows:

$$
\left\langle\xi_{\mathrm{OU}_{i}}(t) \xi_{\mathrm{OU}_{j}}\left(t^{\prime}\right)\right\rangle=\frac{D^{2}}{2 \tau_{d}} \delta_{i, j} e^{-\left|t-t^{\prime}\right| / \tau_{d}}
$$

where the average of the noise term is again zero. This is the socalled Ornstein-Uhlenbeck (OU) noise, which can be obtained by integrating the following ordinary differential equation:

$$
\dot{\xi}_{\mathrm{OU}_{i}}(t)=-\frac{1}{\tau_{d}} \xi_{\mathrm{OU}_{i}}+\frac{1}{\tau_{d}} \xi_{w_{i}}
$$

where $\xi_{w_{i}}$ is a Gaussian white noise, with the correlation defined in Eq. (11).

The main issue is to estimate the value of the noise amplitude $D$ to insert in Eqs. (11) and (12) and of the correlation time of the OU process $\tau_{d}$ to obtain a good reconstruction of the original dynamics. The latter parameter can be straightforwardly quantified from the autocorrelation function decay, in particular we set $\tau_{d}=\tau_{c}=0.1$. For the former quantity, as a first attempt, we set $D$ equal to the time-averaged standard deviation of the fields $\left\langle\sigma_{E}\right\rangle$. However, the quality of the reconstruction was not particularly good and this can be explained by the fact that the fluctuation amplitude is state dependent, as shown in Fig. 1(b). Therefore, we evaluated $\left\langle\sigma_{E}(\bar{E}, \bar{P})\right\rangle$ during simulations of DD systems and we employed these quantities in the stochastic integration of the FC system. In particular, we set $D=\left\langle\sigma_{E}(E, P)\right\rangle$, where $E$ and $P$ are now the values of the fields obtained during the simulation of the FC stochastic system.

We have performed the stochastic integration (see the Appendix for details) by employing extremely small time steps. Such a choice is not due requirements related to the the precision of the integration scheme [in particular, in the whitenoise case the integration would be exact, see (A2)] but to the fact that for the evolution of our system is crucial to detect the spike emissions with extremely accuracy. Therefore, instead of recurring to more elaborate integration schemes [15], we decided to use small integration time steps $h$ in order to accurately determine the threshold crossing of the membrane potentials even in the presence of noise.

\section{LINEAR STABILITY ANALYSIS}

We are not only interested in the reconstruction of the macroscopic and microscopic dynamical features of the DD system via the stochastic approach but also in the reproduction of the linear stability properties of the original model. The latter can be quantified in terms of the Lyapunov spectrum $\left\{\lambda_{i}\right\}$, which can be related to the average growth rates of infinitesimal volumes in the tangent space. The Lyapunov spectrum has been estimated by considering the linearized evolution of the original system and by applying the usual procedure developed by Benettin et al. [16]. Therefore, let us start from the formulation of the linearized evolution of the DD case by differentiating (1) and (3); this reads as:

$$
\begin{aligned}
\delta \dot{E}_{i} & =\delta E_{i}-\alpha \delta P_{i}, \\
\delta \dot{P}_{i} & =-\alpha \delta P_{i}, \\
\delta \dot{v}_{i} & =-\delta v_{i}+g \delta E_{i}, \quad i=1, \ldots, N_{D},
\end{aligned}
$$

where $\left\{\delta E_{i}, \delta P_{i}, \delta v_{i}\right\}$ is a $3 \times N_{D}$ vector in the tangent space. In the following we will limit our analysis to the maximal nonzero Lyapunov exponent.

It should be noted that two discontinuous events are present in the evolution of the original orbit: namely the spike emission, which affects the field variables $\left\{P_{i}\right\}$, and the reset mechanism acting on the membrane potentials $\left\{v_{i}\right\}$. 
However, these discontinuities are not explicitly present in the ordinary differential equations (ODEs) representing the tangent space evolution (14). In the next subsections we will report three different approaches on how to deal with these discontinuities in deterministic systems and the possible extension to stochastic differential equations (SDEs) for two of them. The first approach requires the formulation of the dynamics in terms of an exact event-driven map so we do not see the possibility to extend it to stochastic systems. Instead, the other two methods concern the integration of ODEs with discontinuities and they can be easily extended to SDEs.

\section{A. Linearization of the event-driven map (LEDM)}

This approach can be applied whenever it is possible to write the evolution between two successive events in an exact manner, and the expression (even implicit) of the time interval $\tau(n)$ between two events is known. Here we will focus on the method introduced in Ref. [13] for networks of pulse-coupled LIF neurons. In this case it is possible to write explicitly the linearization of the event-driven map by differentiating Eqs. (4), (5), and (6).

The linearization reads as:

$$
\begin{aligned}
\delta E_{i}(n+1)= & e^{-\alpha \tau(n)}\left[\delta E_{i}(n)+\tau(n) \delta P_{i}(n)\right]-e^{-\alpha \tau(n)} \\
& \times\left[\alpha E_{i}(n)+(\alpha \tau(n)-1) P_{i}(n)\right] \delta \tau(n), \\
\delta P_{i}(n+1)= & e^{-\alpha \tau(n)}\left[\delta P_{i}(n)-\alpha P_{i}(n) \delta \tau(n)\right], \\
\delta v_{i}(n+1)= & e^{-\tau(n)}\left[\delta v_{i}(n)+\left(a-v_{i}(n)\right) \delta \tau(n)\right]+g \delta H_{i}(n) \\
i= & 1, \ldots, N_{D} ; \quad \delta v_{m}(n+1) \equiv 0,
\end{aligned}
$$

where $m$ is the index of the neuron firing at time $t_{n+1}$, while the condition $\delta v_{m}(n+1) \equiv 0$ is a consequence of the Poincaré section we are performing to derive the event-driven map.

The evolution of the LEDM is completed by the expression for $\delta \tau(n)$, which is

$$
\delta \tau(n)=\tau_{v} \delta v_{m}(n)+\tau_{E} \delta E_{m}(n)+\tau_{P} \delta P_{m}(n),
$$

where

$$
\tau_{v}:=\frac{\partial \tau}{\partial v_{m}}, \quad \tau_{E}:=\frac{\partial \tau}{\partial E_{m}}, \quad \tau_{P}:=\frac{\partial \tau}{\partial P_{m}} .
$$

More details regarding this method can be found in Ref. [3] for a DD system.

\section{B. Müller-Dellago-Posch-Hoover (MDPH) method}

A well-known method used for the calculation of Lyapunov exponents for discontinuous flows has been introduced in Refs. [17,18] and it has been recently extended to integrate and fire neural models with refractory periods in Ref. [19] and to piecewise linear models of spiking neurons [20]. Here we will present an application of this method to our DD neuronal model. The approach consists of integrating in parallel the linearized evolution (14) and the ODEs describing the evolution of the orbit, namely (1) and (3), until one of the neurons reaches threshold. At this point the tangent vector value should be updated, due to a discontinuous event, as explained below.

By following the notation used in Ref. [17], let us consider a dynamical system described by a flow equation $\dot{\mathbf{x}}=\mathbf{f}_{\mathbf{1}}(\mathbf{x})$ with a discontinuity defined by some implicit equation in terms of the state variables $l(\mathbf{x})=0$. The evolution at the discontinuity is defined in terms of a function $\mathbf{g}(\mathbf{x})$ mapping the state of the system from the time immediately previous to the discontinuity to the one immediately after, i.e., $\mathbf{x}_{+}=\mathbf{g}\left(\mathbf{x}_{-}\right)$. Finally, let us assume that the dynamics after the discontinuity is ruled by a different flow equation, namely $\dot{\mathbf{x}}=\mathbf{f}_{\mathbf{2}}(\mathbf{x})$.

In this way the evolution in the real space is perfectly defined, while the correction to the tangent space vector $\delta \mathbf{x}$, due to the discontinuity, can be expressed as follows:

$$
\delta \mathbf{x}_{+}=\boldsymbol{G}\left(\boldsymbol{x}_{-}\right) \delta \boldsymbol{x}_{-}+\left[\boldsymbol{G}\left(\boldsymbol{x}_{-}\right) \boldsymbol{f}_{\mathbf{1}}\left(\boldsymbol{x}_{-}\right)-\boldsymbol{f}_{\mathbf{2}}\left(\boldsymbol{x}_{+}\right)\right] \delta t,
$$

where, provided that specific solvability conditions are met [17],

$$
\delta t=-\frac{\boldsymbol{L}\left(\boldsymbol{x}_{-}\right) \delta \boldsymbol{x}_{-}}{\boldsymbol{L}\left(\boldsymbol{x}_{-}\right) \boldsymbol{f}_{\mathbf{1}}\left(\boldsymbol{x}_{-}\right)} .
$$

The notation $\mathbf{x}_{-}\left(\mathbf{x}_{+}\right)$indicates the state of the system right in the moment $t^{*}$ of reaching the discontinuity (just after $t^{*}$ ). Moreover,

$$
\mathbf{L}(\mathbf{x})=\frac{\partial l(\mathbf{x})}{\partial \mathbf{x}} \quad \mathbf{G}(\mathbf{x})=\frac{\partial \mathbf{g}(\mathbf{x})}{\partial \mathbf{x}} .
$$

It is easy to show that for our DD system the flux is given by

$$
\mathbf{f}_{\mathbf{1}}(\mathbf{x})=\mathbf{f}_{\mathbf{2}}(\mathbf{x})=[\dot{\mathbf{E}}, \dot{\mathbf{P}}, \dot{\mathbf{v}}],
$$

and the map at the discontinuity reads as

$$
\begin{aligned}
\mathbf{g}(\mathbf{x})= & {\left[E_{1_{+}}, E_{2_{+}}, \ldots, P_{1_{+}}, P_{2_{+}}, \ldots, v_{1_{+}}, v_{2_{+}}, \ldots, v_{m_{+}}, \ldots\right] } \\
= & {\left[E_{1_{-}}, E_{2_{-}}, \ldots, P_{1_{-}}+\frac{\alpha^{2}}{K} C_{m, 1}, P_{2_{-}}+\frac{\alpha^{2}}{K} C_{m, 2}, \ldots,\right.} \\
& \left.v_{1_{+}}, v_{2_{-}}, \ldots, 0, \ldots\right],
\end{aligned}
$$

where $m$ indicates the neuron firing at time $t^{*}$. Furthermore, the firing condition can be expressed as the scalar function

$$
l(\mathbf{x})=v_{m}-1 .
$$

Therefore, a straightforward calculation gives us the corrections to perform in the tangent space to take into account the firing event at time $t^{*}$ :

$$
\begin{aligned}
& \delta E_{i_{+}}=\delta E_{i_{-}}-C_{m, i} \frac{\alpha^{2}}{K} \delta t, \\
& \delta P_{i_{+}}=\delta P_{i_{-}}+C_{m, i} \frac{\alpha^{3}}{K} \delta t, \\
& \delta v_{m_{+}}=-\dot{v}_{m_{+}} \delta t,
\end{aligned}
$$

with

$$
\delta t=-\frac{\delta v_{m_{-}}}{\dot{v}_{m_{-}}} .
$$

Here $\delta t$ is the (linear) correction to apply to the spike time of the reference orbit to obtain the firing time of the perturbed trajectory. This quantity can be evaluated from the linearization of the threshold condition $v_{m}=1$, and this leads to the following expression:

$$
\delta t=-\frac{1}{\dot{v}_{m_{-}}}\left(\left.\frac{\partial v_{m}}{\partial E_{m}}\right|_{-} \delta E_{m_{-}}+\left.\frac{\partial v_{m}}{\partial P_{m}}\right|_{-} \delta P_{m_{-}}\right),
$$


where all the quantities entering in the right-hand side of the above equation are evaluated exactly at the spiking time.

\section{Olmi-Politi-Torcini (OPT) method}

Recently, another approach has been proposed to deal with the discontinuities occurring in the context of pulse-coupled neural networks [21]. In this context the dynamical evolution in the tangent space between two spike events is ruled by the $3 \times N_{D}$ ODEs reported in (14). Whenever a spike is emitted in the network the tangent space vector components should be updated as follows:

$$
\begin{aligned}
\delta E_{i_{+}} & =\delta E_{i_{-}}+\dot{E}_{i_{-}} \delta t, \\
\delta P_{i_{+}} & =\delta P_{i_{-}}+\dot{P}_{i_{-}} \delta t, \\
\delta v_{i_{+}} & =\delta v_{i_{-}}+\dot{v}_{i_{-}} \delta t,
\end{aligned}
$$

where the expression for $\delta t$ is reported in Eq. (22) and the corrective terms appearing in (24) account for the difference in the spiking times of the perturbed and unperturbed orbit. It is clear that in this case, just after the firing event, the component of the tangent vector corresponding to the membrane potential of the firing neuron is exactly zero, i.e., $\delta v_{m_{+}} \equiv 0$. In this approach, the evolution in tangent space is still performed by taking into account the constraint due to the Poincaré section associated to the event-driven map, meaning that this method is completely analogous to the LEDM.

\section{Comparison of the different methods}

In order to verify the agreement among the different approaches introduced above, we perform numerical estimation of the maximal nonzero Lyapunov exponent by employing such methods for a FC deterministic network. In this case the system is never chaotic and in particular we consider two situations where the microscopic neuronal dynamics is either periodic or quasiperiodic. The first regime corresponds to the so-called splay state (observable for $\alpha=3$ for the chosen parameters) and the latter one to the partially synchronized regime (observable for $\alpha=9$ ). In both cases, it has been shown that the whole branch of the Lyapunov spectrum corresponding to the membrane potentials vanishes as $1 / N^{2}$ in the thermodynamic limit [3]. In order to test for the accuracy of the employed methods, we decided to consider finite-size networks, with $N_{D}=50-200$, where the Lyapunov exponents are extremely small.

It is important to remember that the definition of the LEDM and OPT methods require a Poincaré section. Therefore, one degree of freedom, associated with the motion along the reference orbit, is removed from the dynamical evolution and also the corresponding zero Lyapunov exponent from the Lyapunov spectrum. Conversely, the MDPH method is not based on a Poincaré section. This means that, for a periodic motion, the largest Lyapunov exponent, evaluated with LEDM and OPT methods, corresponds to the second Lyapunov exponent estimated with the MDPH. Similarly, when the neurons evolve quasiperiodically in time, the maximal nonzero Lyapunov exponent obtained with LEDM and OPT is the second one, while being the third one with the MDPH method. In summary, to test the accuracy of the algorithms we compare in the periodic (quasiperiodic) case, the second (third) Lyapunov exponent as obtained by the MDPH method with the first (second) one obtained with the other two methods. We measured these exponents for different system sizes, namely $N_{D}=50,100$, and 200. For all the considered parameter values and system sizes the agreement among the three methods is very good, and the discrepancies among the different estimations are always of the order of $10^{-5}-10^{-6}$, as reported in Table $\mathrm{I}$.

We also tested the three algorithms for a diluted deterministic system where the maximal Lyapunov exponent is definitely positive and its value is two to three orders of magnitude larger than the absolute values of the Lyapunov exponents measured in the nonchaotic situations. In this case to improve the precision of the integration scheme, we employed an event-driven technique where the integration time step is variable and given by the solution of Eq. (5). This implementation allows us to avoid the interpolations required to find the firing times when the integration schemes with

TABLE I. Comparison of the maximal (nonzero) Lyapunov exponents obtained with the three methods introduced in Sec. III, namely the LEDM, OPT, and MDPH methods. Upper panel: For a deterministic FC network in the periodic splay-state regime (left set of parameters) and in the quasiperiodic partially synchronized regime (right set of parameters). Lower panel: For a chaotic DD network in the asynchronous regime (left set of parameters) and in the partially synchronized regime (right set of parameters). In all cases, the system is first relaxed through a transient of $10^{4}$ spikes, after which the Lyapunov exponents are obtained by averaging over a period corresponding to $\simeq 10^{7}$ spike events. The reported errors are calculated as the maximal (absolute) difference between the average of the values obtained with the three methods and each

\begin{tabular}{|c|c|c|c|c|c|c|c|c|}
\hline \multirow[b]{2}{*}{$N_{D}$} & \multicolumn{4}{|c|}{$\alpha=3, g=0.4, a=1.3, K=N_{D}$} & \multicolumn{4}{|c|}{$\alpha=9, g=0.4, a=1.3, K=N_{D}$} \\
\hline & LEDM & OPT & MDPH & Max. Abs. Error & LEDM & OPT & MDPH & Max. Abs. Error \\
\hline 50 & $-1.70 \times 10^{-4}$ & $-1.67 \times 10^{-4}$ & $-1.70 \times 10^{-4}$ & $0.02 \times 10^{-4}$ & $-1.83 \times 10^{-3}$ & $-1.75 \times 10^{-3}$ & $-1.76 \times 10^{-3}$ & $0.05 \times 10^{-3}$ \\
\hline 100 & $-4.25 \times 10^{-5}$ & $-4.30 \times 10^{-5}$ & $-4.38 \times 10^{-5}$ & $0.07 \times 10^{-5}$ & $-4.73 \times 10^{-4}$ & $-4.60 \times 10^{-4}$ & $-4.66 \times 10^{-4}$ & $0.07 \times 10^{-4}$ \\
\hline \multirow[t]{2}{*}{200} & $-1.07 \times 10^{-5}$ & $-1.14 \times 10^{-5}$ & $-9.10 \times 10^{-6}$ & $0.13 \times 10^{-5}$ & $-1.19 \times 10^{-4}$ & $-1.18 \times 10^{-4}$ & $-1.28 \times 10^{-4}$ & $0.06 \times 10^{-4}$ \\
\hline & \multicolumn{4}{|c|}{$\alpha=3, g=0.5, a=1.05, K=0.2 N_{D}$} & \multicolumn{4}{|c|}{$\alpha=9, g=0.5, a=1.05, K=0.2 N_{D}$} \\
\hline$N_{D}$ & LEDM & OPT & MDPH & Max. Abs. Error & LEDM & OPT & MDPH & Max. Abs. Error \\
\hline 200 & $9.4676 \times 10^{-3}$ & $9.4676 \times 10^{-3}$ & $9.4608 \times 10^{-3}$ & $0.45 \times 10^{-5}$ & $2.9515 \times 10^{-1}$ & $2.9515 \times 10^{-1}$ & $2.9514 \times 10^{-1}$ & $0.01 \times 10^{-3}$ \\
\hline
\end{tabular}
single value. The MDPH and OPT estimates are obtained in the upper panel by integrating the system (4) with a fixed time step $h=5 \times 10^{-6}$, while in the lower panel by employing an event-driven integration scheme, where the time step is variable and given by (5). 
a fixed time step are used. Also for the DD systems the discrepancies among the three methods are of order $10^{-5}-10^{-6}$ (as shown in Table I), thus suggesting that these differences are most probably due to the slow convergence of the Lyapunov exponents to their asymptotic value rather than to the precision of the numerical integration. Nonetheless, these results confirm that the three approaches are essentially equivalent for the analysis of deterministic systems.

\section{E. Implementation for SDEs}

Let us explain in detail how we implement the evolution in the tangent space associated to the SDEs Eq. (10). For SDEs the estimation of the maximal Lyapunov exponent has been performed by employing the MDPH and the OPT methods, since the LEDM cannot be used in the case of a stochastic evolution, because it requires an exact knowledge of the next firing time. For white additive noise, the linearized equations for both methods have exactly the same form and they coincide with the expression in absence of noise reported in (14). Notice that in this case we have a common field, and therefore there are only two equations for the evolution of the infinitesimal perturbations $(\delta E, \delta P)$ of the field. The stochastic nature of the process is reflected only in the evolution of the reference orbit around which the linearization is performed. The only approximation we have done in this case is the same adopted during the integration of the real space. Namely, at each firing time the values of the membrane potentials (entering in the tangent space evolution) are simply evaluated as a linear interpolation between the values taken at the time step before and after the firing event and not by employing some accurate stochastic propagator taking in account the presence of absorbing boundaries [15].

In the case of OU noise the situation is more delicate; in particular, the equations for the evolution of the common field correspond to Eqs. (14a) and (14b). On the other hand, the linearized equations for the membrane potentials and the $\mathrm{OU}$ noise terms now read as

$$
\begin{gathered}
\delta \dot{v}_{i}=-\delta v_{i}+g \delta E+g \delta \xi_{\mathrm{OU}_{i}} \\
\delta \dot{\xi}_{\mathrm{OU}_{i}}=-\frac{1}{\tau_{d}} \delta \xi_{\mathrm{OU}_{i}} \quad i=1, \ldots, N_{D} .
\end{gathered}
$$

It is easy to verify via (18) and (19) that the evolution of the Ornstein-Uhlenbeck process does not require extra corrections in correspondence of the firing events when the MDPH method is used, i.e., $\delta \xi_{\mathrm{OU}_{i+}}=\delta \xi_{\mathrm{OU}_{i-}}$.

Instead, with the OPT approach each noise term $\delta \xi_{\mathrm{OU}_{i}}$ should be updated whenever a neuron spikes as follows:

$$
\delta \xi_{\mathrm{OU}_{i_{+}}}=\delta \xi_{\mathrm{OU}_{i_{-}}}+\dot{\xi}_{\mathrm{OU}_{i_{-}}} \delta t
$$

and $\delta t$ is now defined as

$$
\begin{aligned}
\delta t= & -\frac{\delta v_{m_{-}}}{\dot{v}_{m_{-}}} \\
= & \frac{-1}{\dot{v}_{m_{-}}}\left(\left.\frac{\partial v_{m}}{\partial E}\right|_{-} \delta E_{-}+\left.\frac{\partial v_{m}}{\partial P}\right|_{-} \delta P_{-}\right. \\
& \left.+\left.\frac{\partial v_{m}}{\partial \xi_{\mathrm{OU}_{m}}}\right|_{-} \delta \xi_{\mathrm{OU}_{m_{-}}}\right) .
\end{aligned}
$$

\section{RESULTS}

In this section we examine the quality of the reconstruction of the macroscopic and microscopic features and of the stability properties of the DD system in terms of SDEs representing a FC system subject to additive noise. In particular, we consider a massively connected DD network with $K=0.2 N_{D}$ for various system sizes, namely $500 \leqslant N_{D} \leqslant 10000$. We reconstruct the dynamics of these systems by employing a small FC stochastic system of size $N_{S}=100$, as we have verified that finite-size effects are of limited relevance for FC systems. For each size of the DD system, we employ as noise amplitude in the stochastic FC system the standard deviation of the fluctuations of the corresponding DD fields. In particular, for the chosen setup (massively connected), as the system size of the DD increases the amplitude of the fluctuations of the fields decreases, vanishing in the thermodynamic limit [as shown in Fig. 1(c)].

\section{A. Macroscopic and microscopic dynamics}

In order to test for the quality of the reconstruction of the macroscopic dynamics, we proceed to calculate the PDFs of the common field variables $E$ and $P$ in the FC setup and compare them with the histograms of the average fields $\bar{E}$ and $\bar{P}$ as obtained in the DD case. These are reported in Figs. 2(a) and 2(d) for two system sizes of the diluted system, namely $N_{D}=500$ and $N_{D}=5000$. The agreement between the original PDFs and the reconstructed ones improve by passing from white to colored noise. In particular, this is evident for the $F(E)$, since in the case of white noise these distributions present oscillations which are absent in the original ones. The origin of these oscillations can be ascribed to the fact that in presence of white noise of equal amplitude along the whole macroscopic orbit the field can be driven occasionally far from the original attractor.

When the colored noise is employed one observes a better overall reconstruction of the macroscopic attractors with respect to white noise. This is evident from Figs. 2(e) and 2(f): The attractors obtained with OU noise show fewer deviations from the DD attractor with respect to the white-noise case, in particular around the maximal $\bar{P}$. This is confirmed by considering the evolution in time of the original and reconstructed fields. The time traces of the fields are compared in Figs. 2(g) and 2(h) by matching the time occurrence of the first maximum of each field. As one can see from the figure the OU reconstructed field follows reasonably well the original evolution, at least in the considered time window, while the field of the system driven by white noise shows, already after few oscillations, a delay or advance with respect to the original one.

To render more quantitative this analysis, we have measured the average oscillation period of the field $\left\langle T_{E}\right\rangle$ for various system sizes $N_{D}$ of the DD networks and for the corresponding stochastic reconstructions with white and OU noise. The results for all the considered system sizes are displayed in Fig. 2(i). In the DD case $\left\langle T_{E}\right\rangle$ increases for increasing $N_{D}$ and tends towards the corresponding deterministic FC value (dot-dashed line in the figure), this value will be reached in the thermodynamic limit, as expected [3]. Both the stochastic 

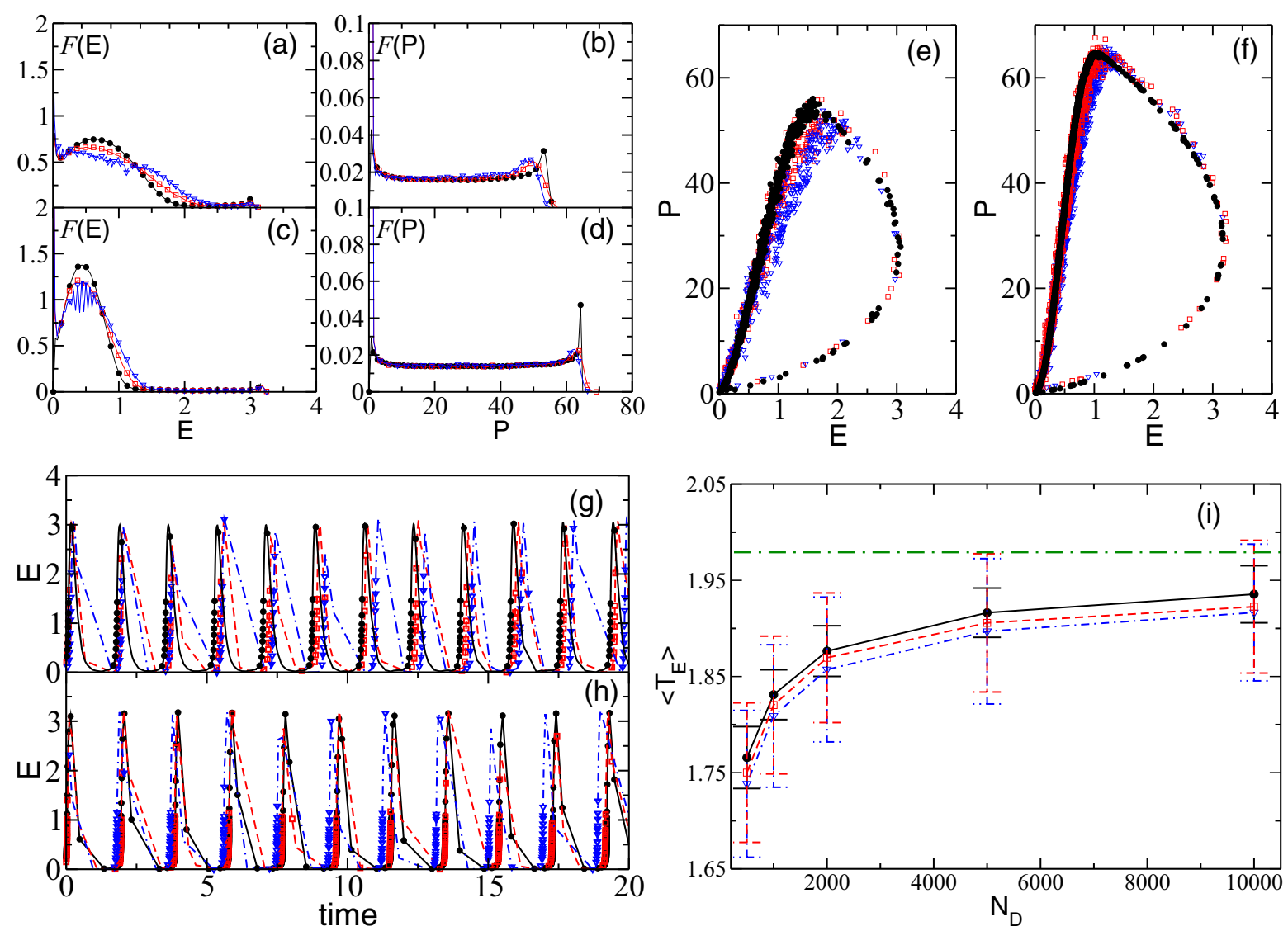

FIG. 2. (Color online) Reconstruction of the macroscopic dynamics of the DD system (black filled circles) in terms of white (blue empty triangles) and colored noise (red empty squares). [(a)-(d)] Histograms of the macroscopic fields $E$ and $P$; [(e) and (f)] macroscopic attractors; [(g)-(h)] time traces of the field $E$; (i) average period of the field $\left\langle T_{E}\right\rangle$ as a function of the system size $N_{D}$ of the DD system. Panels (a), (b), (e), and (g) refer to $N_{D}=500$, while panels (c), (d), (f), and (h) refer to $N_{D}=5000$. In (g) and (h) the time traces have been shifted in order to ensure for the coincidence of the time of occurrence of the first maximum in each trace; moreover, the colored (white) noise has been identified with a dashed (dashed-dotted) line for the sake of visualization in $(\mathrm{g}),(\mathrm{h})$, and (i). The periods reported in (i) have been obtained by measuring the time lapse between two consecutive maxima, the number of samples used for the calculation of $T_{E}$ is 5000 data points, and the (green) dot-dashed line is the field period in a corresponding FC deterministic network equal to 1.98. Reconstructed dynamics have been obtained with $N_{S}=100$ with an integration step $h=5 \times 10^{-6}$. Other parameters are as in Fig. 1 .

estimations slightly underestimate the DD value; however, while the periods obtained by employing OU noise exhibit errors with respect to the DD values of the order $\simeq 0.4-0.9 \%$, the errors made with the white-noise reconstruction are usually larger, namely between 1.0 and $1.5 \%$.

Let us now examine the microscopic dynamics of the DD system. This is quite peculiar for the chosen parameters, corresponding to quasiperiodic evolution of the membrane potentials of the single neurons. Indeed, the single-neuron motion become exactly quasiperiodic only in the thermodynamic limit, where the regular FC dynamics is recovered. For DD systems, as the ones here examined, the neurons evolve on an almost quasiperiodic orbit, apart small chaotic fluctuations. These motions can be analyzed by considering the interspike interval (ISI) of the single cell, in particular, we will estimate the associated PDF $F$ (ISI) as well as the first return maps for the ISIs of the single neurons.

The distributions $F$ (ISI) are reported in Figs. 3(a) and 3(c) for the same level of dilution and two different system sizes, namely $N_{D}=500$ and $N_{D}=5000$. The $F($ ISI) are defined over a finite range of values, corresponding to the values taken by the ISIs during the neuron evolution. By increasing $N_{D}$, which corresponds to have smaller fluctuations $\left\langle\sigma_{E}\right\rangle$, the $F$ (ISI) exhibit a sharper peak at large ISI and at the same time the return map appears to better approach a closed line, as expected for quasiperiodic motions [see Figs. 3(b) and 3(d)].

As far as the corresponding SDEs are concerned, the stochastic reconstruction is fairly good for the $F$ (ISI), despite the fact that the distributions are now covering a slightly wider range with respect to the original PDFs. The reconstructed return maps are noisy closed curves following closely the DD ones. By increasing $N_{D}$ the reconstruction improves both with white and colored noise [as shown in Figs. 3(a)-3(d)]; however, it is difficult to distinguish among the two approaches relying on these indicators. Therefore, we have measured the average ISI for different $N_{D}$ in the DD case and for the corresponding stochastic dynamics. The results are reported in Fig. 3(e). In the DD case, the $\langle$ ISI $\rangle$ increases with $N_{D}$ approaching the FC deterministic limit (green dot dashed line in the figure). The reconstructed $\langle$ ISI $\rangle$ are slightly underestimating the deterministic results; however, they reproduce quite closely the deterministic values. From the figure it is clear that the $\mathrm{OU}$ 

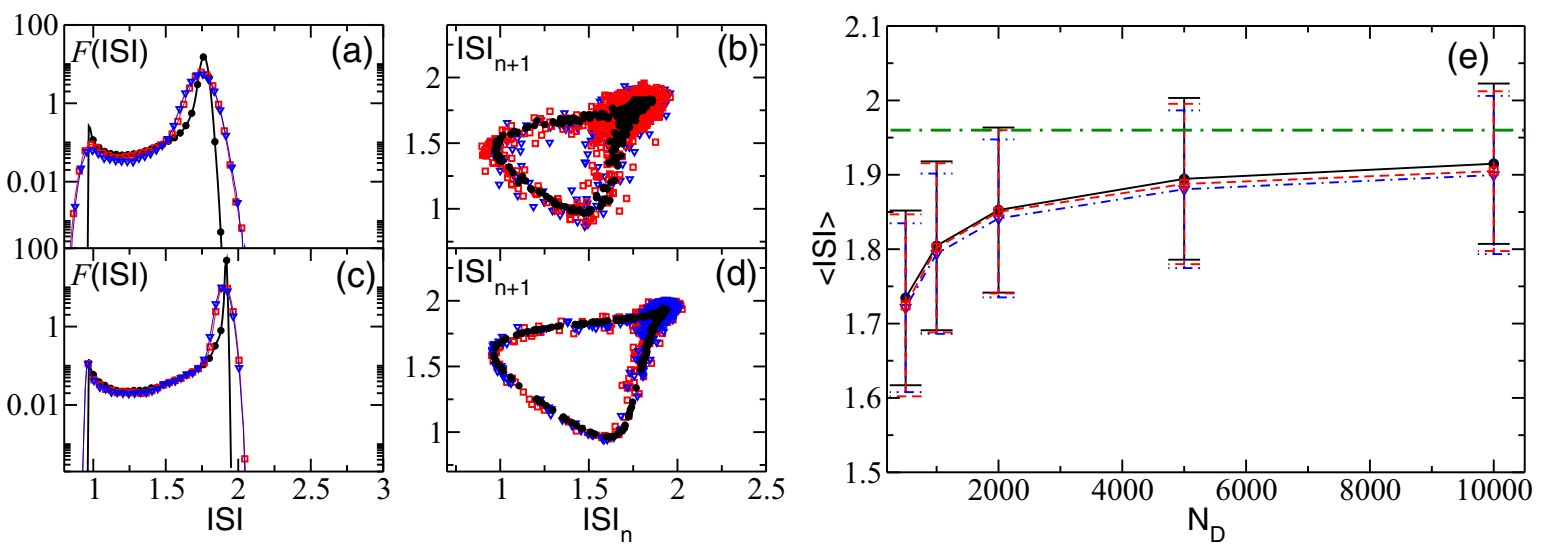

FIG. 3. (Color online) Reconstruction of the microscopic dynamics of the DD system (black filled circles) in terms of white (blue empty triangles) and colored noise (red empty squares). [(a) and (c)] PDF F (ISI) of the ISIs; [(b) and (d)] ISIs return maps. Panels (a) and (b) refer to $N_{D}=500$, while panels (c) and (d) to $N_{D}=5000$. (e) Average ISI as a function of the DD system size $N_{D}$, the thick (green) dot-dashed line refers to the asymptotic value of the average ISI in the corresponding FC deterministic set up, equal to 1.96 time units. Also the colored (white) noise has been identified with a dashed (dashed-dotted) line for the sake of visualization in (e). The reconstructed dynamics are obtained with $N_{S}=100$ by employing an integration step $h=5 \times 10^{-6}$. The other parameters are as in Fig. 1 .

reconstruction represents a better approximation of the DD results for all the considered $N_{D}$, with errors ranging from 0.1 to $0.6 \%$, with respect to the white noise results exhibiting discrepancies between 0.6 and $0.8 \%$ with respect to the DD values.

From the analysis of the macroscopic and microscopic features we can conclude that the stochastic reconstruction improves by passing from white to Ornstein-Uhlenbeck noise. This is particularly evident for the field $E$. The reason for this can be understood by considering the evolution of the macroscopic field: $E$ displays a rapid rising phase of duration $\simeq 0.1-0.2$ followed by a relaxation period $\simeq 0.9 \times T_{E}$ [as shown in Figs. 2(g) and 2(h)]. Therefore, in order to properly reproduce this fast rise induced by the firing of the most part of the neurons in the network, the time correlation of the fluctuations (on a time scale $\tau_{c} \simeq 0.1$ ) should be taken into account.

We have also considered the so-called sparse limit; in particular, we examined the DD networks obtained by fixing $K=100$ and by increasing the system size from $N_{D}=500$ to 10000 . As can be appreciated from Fig. 4, the quality of the stochastic reconstruction obtained at the microscopic and macroscopic level is comparable with the one obtained for the massively connected networks. Thus suggests that our approach holds also for extremely diluted systems.

On the other hand, we have verified that the proposed stochastic reconstruction fails whenever the system does not support a collective dynamical evolution, in the specific case this corresponds to partial synchronization. In fully coupled excitatory systems partial synchronization emerges only in a certain range of parameters, otherwise one observe asynchronous dynamics [2]. However, even within this range of parameters, the diluted system cannot sustain the collective motion if the average in-degree becomes too small $[5,6]$. This is what happens also for the present choice of parameters for $K<40$; notice that this critical connectivity is strongly dependent on the model parameters but not on $N_{D}$ whenever the system size becomes sufficiently large (for more details see Ref. [5]).

\section{B. Lyapunov exponents}

As already mentioned, the system is chaotic for the DD system and the largest Lyapunov exponent vanishes in the
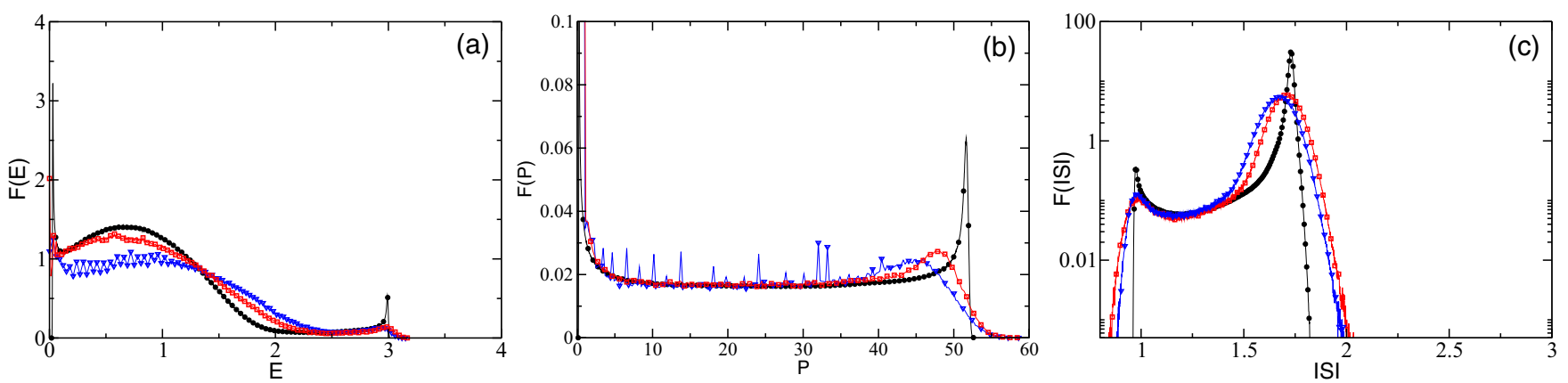

FIG. 4. (Color online) Reconstruction of the microscopic and macroscopic dynamics for a representative size of the DD system in the limit of large dilution. From left to right, reconstruction of the distributions of the instantaneous fields $E, P$ and of the single-neuron ISIs. The considered DD network (black filled circles) has a size $N_{D}=10000$ and an in-degree $K=100$, while the reconstructed dynamics, both for the white noise (blue empty triangles) and the colored noise (red empty squares), are obtained with $N_{S}=100$, integrated with a step $h=5 \times 10^{-6}$. Other parameters are as in Fig. 1. 

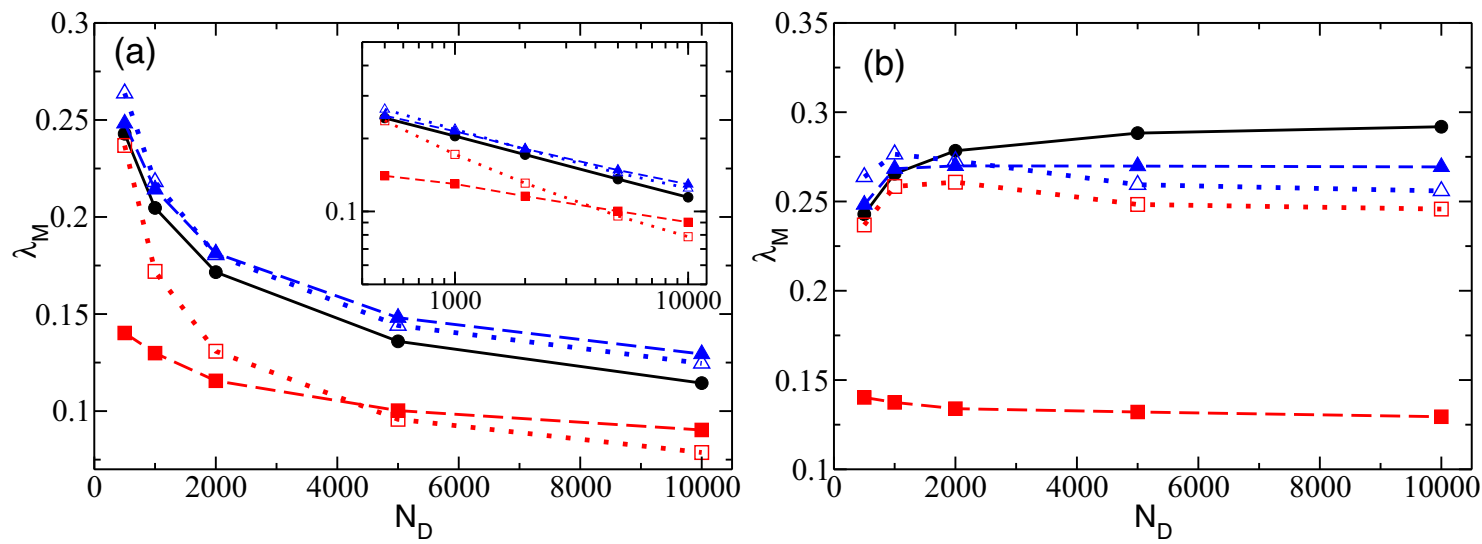

FIG. 5. (Color online) Maximal Lyapunov exponent $\lambda_{M}$ as a function of the system size $N_{D}$ for the DD case (black filled circles and solid line) and the corresponding stochastic reconstructions evaluated with the MDPH method (red squares) and the OPT approach (blue triangles). The stochastic results are reported for white (empty symbols and dotted lines) and Ornstein-Uhlenbeck (filled symbols and dashed lines) noise. In panel (a) we report the results for massively connected networks, where $K=0.2 \times N_{D}$ and in (b) for sparse networks where $K=100$ independent from the value of $N_{D}$. The inset of panel (a) shows in double logarithmic scale $\lambda_{M}$ versus $N_{D}$ for the massively connected networks. Reconstructed dynamics obtained with $N_{S}=100$ and an integration step of $h=5 \times 10^{-6}$. In all cases, the system is relaxed during a transient of $10^{6}$ spikes and the Lyapunov exponents are calculated by integrating the tangent space for a period corresponding to $10^{7}-10^{8}$ spikes. Other parameters are as in Fig. 1.

thermodynamic limit following a power-law decay with $N_{D}$ [5]. In particular, in the considered case we observe a decay $\lambda_{M} \propto N^{-\gamma}$ with $\gamma \simeq 0.25$ [as shown in the inset of Fig. 5(a)], which corresponds to a divergence of the maximal Lyapunov exponent with the averaged field fluctuations given by $\lambda_{M} \propto$ $\left\langle\sigma_{E}\right\rangle^{0.43}$. On the other hand, the FC deterministic counterpart exhibits a perfectly regular dynamics for any system size. Our aim is to reproduce the level of chaoticity present in the DD system by perturbing stochastically the FC system with noise terms whose amplitude corresponds to that of the fluctuations of the deterministic fields $\left\{E_{i}\right\}$, thus demonstrating that these fluctuations are at the origin of the chaotic behavior.

The maximal Lyapunov exponents in the DD case have been estimated by employing the LEDM method, while for the stochastic reconstructions we have used the MDPH and OPT methods with white and OU noise. As is evident from Fig. 5(a), the MDPH largely fails in reproducing the DD data, both for white and colored noise, apart for the smallest system size here considered (namely $N_{D}=500$ ) and white noise. On the other hand, the OPT approach works quite well both with white and OU noise over all the examined ranges of network sizes. The values obtained from the reconstructed dynamics are always larger than the DD values, but while in the OU case the error in the estimation increases with $N_{D}$ and ranges from $2 \%$ at $N_{D}=500$ to $13 \%$ at $N_{D}=10000$, for the SDEs with white noise the error is of the order of $\simeq 5-9 \%$ and it seems not to depend on the considered system size. Furthermore, the OPT estimation of the maximal Lyapunov exponent is able to recover the correct power-law scaling with $N_{D}$, in particular in the white- (OU) noise case we have found an exponent $\gamma \simeq 0.25(\gamma \simeq 0.22)$. The exponent found for the white-noise reconstruction coincides with the deterministic value.

Furthermore, we have verified that for sparse networks all the considered stochastic reconstructions show that the maximal Lyapunov exponent saturates to a constant value in the thermodynamic limit [see Fig. 5(b)]. As expected, due to the fact that the fluctuations of the fields remain essentially constant by increasing $N_{D}$, since $\left\langle\sigma_{E}\right\rangle \propto K^{-1 / 2}$ [12]. However, as can be appreciated from Fig. 5(b), the OPT estimation with OU noise provides in this case the best reconstruction of $\lambda_{M}$ obtained for the DD system, with a maximal discrepancy of $\simeq 7 \%$ by fixing $K=100$ and by varying $500 \leqslant N_{D} \leqslant 10000$.

A possible explanation for the worse performances of the MDPH method with respect to the OPT one for the estimation of $\lambda_{M}$ for a stochastic process with discontinuities relies on the definition and implementation of the method. As shown in Eq. (21c) and Eq. (22) the corrections to be applied at each firing event depends only on the value of the derivative of the membrane potential of the firing neuron estimate just before $\left(\dot{v}_{m_{-}}\right)$and after $\left(\dot{v}_{m_{+}}\right)$the event. These quantities depend on the actual value of the membrane potential at threshold and reset; as a matter of fact we have assumed that these values are not affected by noise. Maybe this assumption is too restrictive; however, no better results have been obtained by the inclusion of stochastic terms. Instead, for the OPT approach the occurrence of a spike is taken into account by modifying the values of the linearized variables in the tangent space on the basis of the time derivatives of the corresponding variables (in the real space) evaluated just before the spike emission [see Eq. (24)]. These time derivatives have been estimated as a linear interpolation between the values taken at the integration step immediately before and after the spike; therefore, in their evaluation, the stochastic evolution is somehow taken in account.

\section{v. CONCLUSIONS}

We have shown that the effect of the randomness in the distribution of the connections among neurons can be reproduced in terms of a perfectly regular (FC) network, where an additive noise term is introduced in the evolution equations for the membrane potentials. This suggests that 
either noise or dilution can lead to similar effects on the network dynamics (at least) in systems exhibiting collective oscillations. These results open new interesting directions for the study of the macroscopic activity of large sparse (neural) networks, which can be mimicked in terms of few collective noisy variables. Furthermore, our analysis has revealed that the stochastic approach is extremely convenient from a computational point of view, since it allows us to mimic the dynamics of a deterministic system with $3 \times N_{D}$ variables by employing $N_{S}+2$ variables, where $N_{S}=100$, irrespectively of the size of the original network. We have also employed larger $N_{S}$, namely $N_{S}=400$, without observing any substantial improvement with respect to the smaller size.

We have also discussed and critically re-examined the existing methods to evaluate Lyapunov exponents for deterministic dynamical models with discontinuities and specifically for pulse-coupled systems. In particular, we have introduced a novel method to estimate stochastic Lyapunov exponents for dynamical systems with discontinuities. Furthermore, we have applied this novel approach in order to give convincing evidence that the fluctuations of the macroscopic variables acting on the membrane potentials are indeed responsible for the presence of chaotic activity in diluted networks of LIF excitatory neurons exhibiting collective oscillations. This is not obvious for any kind of LIF circuits, and recent works [22-25] have shown the existence of linearly stable dynamics in sparse inhibitory networks where the fluctuations of the currents are responsible for the irregular activity of the system, in the absence of chaotic motion.

The approach presented here appears to work reasonably well in the presence of collective oscillations in the macroscopic field (i.e., partial synchronization in the network), while we have verified that, when the global activity is asynchronous, the reconstructions do not perform equally well. The origin of this discrepancy can be traced back to the fact that the fluctuations of the fields are, in the asynchronous situation, almost periodic with decorrelation times $\mathcal{O}\left(10^{2}\right)$. Such slow decorrelation demands for more refined treatment of the noise term, like, e.g., by considering harmonic noise terms [26]. Furthermore, a higher fidelity is needed in the tangent space reconstruction since the maximal Lyapunov exponent is, in this case, two orders of magnitude smaller than for the partially synchronized dynamics.

Our approach can be considered a sort of stochastic mean-field version of the original system; in this regard it should be mentioned that in recent works, the reconstruction of the dynamics of a diluted neural model quite similar to the one analyzed here has been successfully attempted by employing a deterministic heterogeneous mean-field (HMF) approach [27]. The HMF amounts to introduce mean-field variables associated to equivalence classes of neurons with the same in-degree, but it still maintains the heterogeneous character of the diluted system, thus not allowing us to clearly single out the source of the chaotic activity.

\section{ACKNOWLEDGMENTS}

This work has been supported by the European Commission under the program "Marie Curie Network for Initial Training," through Project No. 289146, "Neural Engineering
Transformative Technologies (NETT).”D.A.-G. also acknowledges the partial support provided by "Departamento Adminsitrativo de Ciencia Tecnologia e Innovacion-Colciencias" through the program "Doctorados en el exterior-2013." The authors also thank Professor André Longtin for very fruitful discussions and Dr. Mario Mulansky and Dr. Stefano Lepri for a careful reading of the manuscript prior to submission.

\section{APPENDIX: INTEGRATION OF THE STOCHASTIC DIFFERENTIAL EQUATIONS}

Let us now examine how we can perform the integration of the SDEs (10) for the white and OU noise. The integration of the ODEs for the fields $E$ and $P$ can be performed without any approximation analogously to what done for the event-driven map (4a) and (4b), since their evolution is completely deterministic. The integration of the equation for the membrane potential (10c) is instead performed in two steps; first, the deterministic part is integrated from time $t$ to $t+h$ as

$$
\mathcal{F}_{i}(v)=v_{i}(t) \mathrm{e}^{-h}+a\left(1-\mathrm{e}^{-h}\right)+g H(h) .
$$

Then the stochastic part is considered, for the white-noise case, due to the linearity of the SDE the stochastic process can be integrated exactly [15], and the solution reads as

$$
v_{i}(t+h)=\mathcal{F}_{i}(v)+D \sqrt{\frac{1}{2}\left(1-\mathrm{e}^{-2 h}\right)} \eta_{i}(t)
$$

Here the stochastic variable $\eta_{i}(t)$ is a spatiotemporal uncorrelated random number, normally distributed with zero average and unitary variance.

For the colored noise, instead, the integration of the SDE with accuracy $\mathcal{O}\left[h^{2}\right]$ leads to the following set of equations [28]:

$$
\begin{aligned}
\xi_{\mathrm{OU}_{i}}(0) & =\frac{D}{\sqrt{\tau_{d}}} \eta_{i}(0), \\
\xi_{\mathrm{OU}_{i}}(t+h) & =\xi_{\mathrm{OU}_{i}}(t) e^{-h / \tau_{d}}+D \sqrt{\frac{1-e^{-2 h / \tau_{d}}}{\tau_{d}}} \eta_{i}(t), \\
v_{i}(t+h) & =\mathcal{F}_{i}(v)+h \xi_{\mathrm{OU}_{i}}(t) .
\end{aligned}
$$

The integration is performed with a constant time step $h \simeq$ $10^{-5}-10^{-6}$. In particular, we integrate exactly the equations for the field variables $E$ and $P$ for a time interval $h$, while the membrane potential is evaluated employing the stochastic propagators reported in Eqs. (A2) or (A3), depending on whether we consider white or OU noise. Whenever the membrane potential of one neuron overcomes threshold, we evaluate the crossing time $t^{*}$ and the values of all the membrane potentials at $t^{*}$ via a linear interpolation. We then restart the integration with the values of the field variables and of the membrane potentials evaluated at $t^{*}$ after resetting the potential of the neuron which has just fired. 
[1] L. F. Abbott and C. van Vreeswijk, Phys. Rev. E 48, 1483 (1993).

[2] C. van Vreeswijk, Phys. Rev. E 54, 5522 (1996).

[3] S. Olmi, R. Livi, A. Politi, and A. Torcini, Phys. Rev. E 81, 046119 (2010).

[4] D. Golomb, D. Hansel, and G. Mato, in Handbook of Biological Physics (Elsevier Science, New York, 2001), Vol. 4, p. 887.

[5] L. Tattini, S. Olmi, and A. Torcini, Chaos 22, 023133 (2012).

[6] S. Luccioli, S. Olmi, A. Politi, and A. Torcini, Phys. Rev. Lett. 109, 138103 (2012).

[7] C. J. Tessone, C. R. Mirasso, R. Toral, and J. D. Gunton, Phys. Rev. Lett. 97, 194101 (2006).

[8] C. J. Tessone, A. Scirè, R. Toral, and P. Colet, Phys. Rev. E 75, 016203 (2007).

[9] B. Huberman, J. Crutchfield, and N. Packard, Appl. Phys. Lett. 37, 750 (1980).

[10] J. B. Gao, S. K. Hwang, and J. M. Liu, Phys. Rev. Lett. 82, 1132 (1999).

[11] B. Dennis, R. A. Desharnais, J. Cushing, S. M. Henson, and R. Costantino, Oikos 102, 329 (2003).

[12] S. Olmi and A. Torcini, Scholarpedia 8, 30928 (2013).

[13] R. Zillmer, R. Livi, A. Politi, and A. Torcini, Phys. Rev. E 76, 046102 (2007).

[14] R. Albert and A.-L. Barabási, Rev. Mod. Phys. 74, 47 (2002).

[15] R. Mannella, Phys. Lett. A 254, 257 (1999).
[16] G. Benettin, L. Galgani, A. Giorgilli, and J.-M. Strelcyn, Meccanica 15, 9 (1980).

[17] P. C. Müller, Chaos. Soliton. Fract. 5, 1671 (1995).

[18] C. Dellago, H. A. Posch, and W. G. Hoover, Phys. Rev. E 53, 1485 (1996).

[19] D. Zhou, Y. Sun, A. V. Rangan, and D. Cai, J. Comput. Neurosci. 28, 229 (2010).

[20] S. Coombes, R. Thul, and K. Wedgwood, Physica D 241, 2042 (2012).

[21] S. Olmi, A. Torcini, and A. Politi, Front. Comput. Neurosci. 8, 8 (2014).

[22] R. Zillmer, R. Livi, A. Politi, and A. Torcini, Phys. Rev. E 74, 036203 (2006)

[23] S. Jahnke, R.-M. Memmesheimer, and M. Timme, Phys. Rev. Lett. 100, 048102 (2008).

[24] M. Monteforte and F. Wolf, Phys. Rev. X 2, 041007 (2012).

[25] D. Angulo-Garcia and A. Torcini, Chaos. Soliton. Fract. 69, 233 (2014).

[26] A. Neiman and L. Schimansky-Geier, Phys. Rev. Lett. 72, 2988 (1994).

[27] M. di Volo, R. Burioni, M. Casartelli, R. Livi, and A. Vezzani, Phys. Rev. E 90, 022811 (2014).

[28] M. San Miguel and R. Toral, in Instabilities and Nonequilibrium Structures VI (Springer, Berlin, 2000), pp. 35-127. 